\title{
Detecting abnormal ozone measurements with a deep learning-based strategy
}

\author{
Fouzi Harrou, Member, IEEE, Abdelkader Dairi, Ying Sun, Farid Kadri
}

\begin{abstract}
Air quality management and monitoring are vital to maintaining clean air, which is necessary for the health of human, vegetation, and ecosystems. Ozone pollution is one of the main pollutants that negatively affect human health and ecosystems. This paper reports the development of an unsupervised and efficient scheme to detecting anomalies in unlabelled ozone measurements. This scheme combines a Deep Belief Networks (DBN) model and a one-class support vector machine (OCSVM). The DBN model accounts for nonlinear variations in the ground-level ozone concentrations, while OCSVM detects the abnormal ozone measurements. The performance of this approach is evaluated using real data from Isère in France. We also compare the detection quality of $\mathrm{DBN}$-based detection schemes to that of deep stacked auto-encoders, Restricted Boltzmann Machinesbased OCSVM and DBN-based clustering procedures (i.e., Kmeans, Birch and Expectation Maximization). The results show that the developed strategy is able to identify anomalies in ozone measurements.
\end{abstract}

Index Terms-Air quality, learning, OCSVM, Ozone, DBNs, statistical monitoring, anomaly detection.

\section{INTRODUCTION}

$\mathbf{T}$ $\mathrm{O}$ achieve acceptable air quality and better understand air pollution phenomena, investigations on the health influences of ambient air pollution have attracted numerous efforts throughout the last few decades [1]-[6]. The surveillance of atmospheric pollution is of great importance due to the negative effects of pollution on health of human and the ecosystem [7], [8]. The high levels of air pollution have become an important issue and can leads to serious impacts on the human health [2], [9], [10]. Pollution from ozone $\left(\mathrm{O}_{3}\right)$ formed in the troposphere is a growing issue in industrialized countries, mainly due to its participation in greenhouse gas emissions [7]. Accordingly, air quality management and monitoring are key to assuring a healthy living environment [11].

Air quality is a key challenging problem increasingly gaining attention worldwide [12], [13]. Ground-level ozone $\left(\mathrm{O}_{3}\right)$, which is an important pollutant that affect everyones health, is basically generated by a chemical reaction of nitrogen and carbon-based compounds discharged in vehicle exhausts [12], [13]. The reactions creating ozone are favored

F. Harrou and Y. Sun are with King Abdullah University of Science and Technology (KAUST) Computer, Electrical and Mathematical Sciences and Engineering (CEMSE) Division, Thuwal, 23955-6900, Saudi Arabia e-mail: fouzi.harrou@kaust.edu.sa

A. Dairi is with Computer Science Department, University of Oran 1 Ahmed Ben Bella , Algeria Street El senia el mnouer bp 31000 Oran, Algeria. E-mail: dairi.aek@gmail.com

F. Kadri is with Sopra Steria Group, Big Data \& Data Analytics Team, 31770 Colomiers, France. E-mail: farid.kadri@soprasteria.com by bright sunlight and usually result in a severe air quality issue named photochemical smog [14]-[16]. Thus, there is a need for systematic approach to detecting abnormal ozone levels, which provides pertinent information for enhancing air quality management or providing useful information for rapid decision-making, such as warning the public of harmful ozone levels or checking the sensors in case a technical problem is causing anomalies. [17]-[19]. This paper presents an flexible monitoring scheme to detect abnormal ozone data.

Recently, deep learning-based feature extraction mythologies turn out to play a considerable role in the literature [20][24]. As a matter of fact, deep learning methods were designed to model complex systems with flexibility, simplicity, and strength using series of multilayer architectures. Fo instance, they are used to enhance intelligent transportation systems [22], [25], health informatics [23], human action recognition [26], detection of the cerebral microbleed voxels [20], classification of hearing loss images [21], and in fingerprints indoor positioning via WIFI [27]. Due to its broad applications, the Deep Belief Networks (DBNs) models, have received much attention from researchers recently. However, to the best of our knowledge, the DBNs models have not been employed for ozone pollution monitoring.

Constructing a fast and precise model of ozone variation is very challenging mainly because its mechanisms of production in the troposphere are complex and there are measurement uncertainties in all the parameters involved [28], [29]. To bypass this difficulty, in this study we use DBN to model the variability in ozone data. It shows a good performance in learning layer-by-layer complex nonlinearity [30]. The purpose is to design a deep learning-based methodology capable to detect abnormal ozone measurements. Basically, this method combines a DBN model with one-class support vector machine (OCSVM), to simultaneously take benefits of a DBN model in extracting features from high dimensional data and the anomaly detection capability of OCSVM. We assess our approach via data obtained from the Isère region in France. We also compare the detection quality of DBN-based detection schemes to that of deep stacked auto-encoders (DSA) and Restricted Boltzmann Machines-based OCSVM and other clustering procedures.

The study site and data collection are briefly described in Section II. Section III reviews the DBN model and the OCSVM scheme. Then, the DBN modeling approach and the OCSVM procedure are introduced in Section III. Section IV presents the designed monitoring technique. In Section V, we 
assess the efficiency of the developed approach using via real data and conclusions are presented in Section VI.

\section{STUDIED AIR QUALITY MONITORING NETWORK}

In this paper, we monitor ozone measurements from Isère, France (see Figure 1). Atmo Auvergne-Rhône-Alpes is the establishment in charge of supervision of air quality in this region. Hourly ozone data are collected via 14 stations dispersed throughout the region (see Table I). Each station consists of a room with many analyzers (see Figure 2). The ambient air is taken in from the roof of each measuring station and pumped to the measuring instruments located inside the station. Each analyzer is used to a specific pollutant (e.g., ozone). The ambient air is continuously analyzed 24 hours a day and 7 days a week. The data measured at the network stations are transmitted by telephone line to a central computer, stored in a local database, processed statistically, and validated manually every day by the Atmo team. The validated data are then sent to a national database.

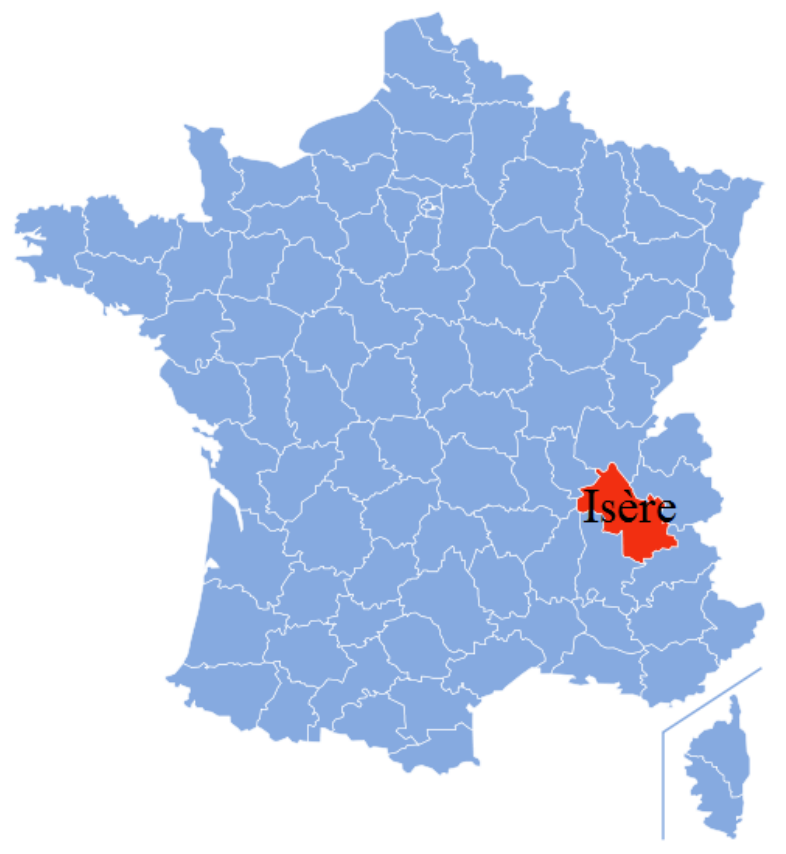

Fig. 1. Location of Isère in France.

The spatial distribution of measurement stations is shown in Figure 3.

\section{PReliminary MATERIAL}

Here, we briefly describe the DBN model and the OCSVM scheme, which are used to construct the proposed detection approach.

\section{A. Deep Belief Networks}

DBNs models can basically be obtained by piling succession of many Restricted Boltzmann Machines (RBMs). These latter represent stochastic neural networks (see Figure 5), which can be viewed as a special case of Boltzmann machines (BM). They are efficient to estimate the probability distribution of

\begin{tabular}{|l|l|l|l|}
\hline Station & Type & $\mathrm{O}_{3}$ & Symbol \\
\hline Vienne Centre & Urban & $12-08-2003$ & S1 \\
\hline Les Roches de Condrieu ZI & Peri-urban & $01-07-1998$ & S2 \\
\hline Roussillon & Urban & $16-06-1994$ & S3 \\
\hline Sud roussillonnais / Sablons & Peri-urban & $04-09-2003$ & S4 \\
\hline Bourgoin-Jallieu & Urban & $15-07-2004$ & S5 \\
\hline Plateau de Bonnevaux & Rural & $24-12-2013$ & S6 \\
\hline Voiron Urbain & Urban & $24-11-2010$ & S7 \\
\hline Est grenoblois / Grésivaudan & Peri-urban & $09-01-2008$ & S8 \\
\hline Fontaine les Balmes & Urban & $29-12-1999$ & S9 \\
\hline Grenoble Caserne de Bonne & Urban & $08-03-2016$ & S10 \\
\hline Saint-Martin d'Heres & Urban & $25-08-1998$ & S11 \\
\hline Grenoble les Frenes & Urban & $12-02-2001$ & S12 \\
\hline Sud grenoblois / Champ sur Drac & Peri-urban & $26-01-1988$ & S13 \\
\hline Sud grenoblois / Vif & Peri-urban & $16-01-2008$ & S14 \\
\hline \multicolumn{2}{|l|}{ TABLE I } & & \\
\hline
\end{tabular}

MEASUREMENT STATIONS IN ISÈRE REgION.

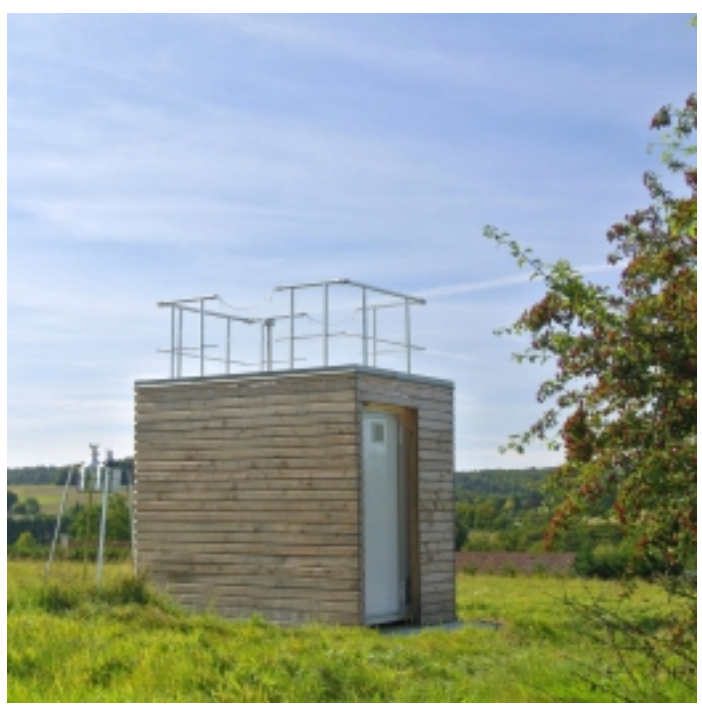

Fig. 2. Measurement station.

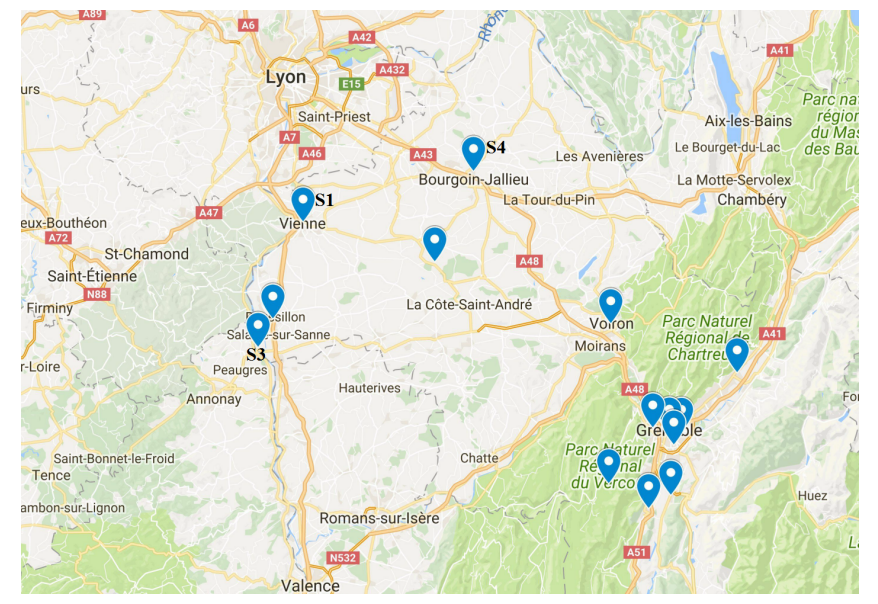

Fig. 3. Network of measurement stations for the Isère region.

input data. RBMs are generative models that can be used to generate new data by sampling from the model. They are defined with the restriction: no interaction between hidden variables, i.e., neurons belonging to the same layer are not connected. However, a full connection mode exists with the 


\section{Restricted Boltzmann Machines}

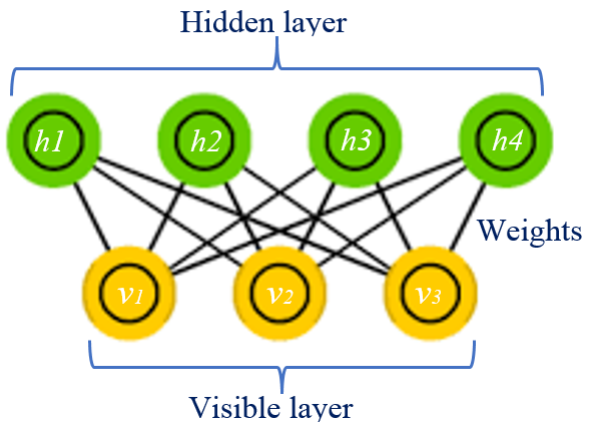

Fig. 4. Structure of an RBM model.

neurons in the other layer. RBMs are undirected graphs with a simple structure composed of a visible layer $v$ representing the input data (observed) and hidden (or latent) layer corresponding to the feature detectors. The interaction between the visible and hidden units $(v \Leftrightarrow h)$ has an energy expressed by the following energy function [31]:

$$
\operatorname{Energy}(v, h)=-\sum_{i=1}^{m} \sum_{j=1}^{n} W_{i j} v_{i} h_{j}-\sum_{i=1}^{m} b_{i} v_{i}-\sum_{j=1}^{n} c_{j} h_{j},
$$

where $W_{i j}$ is the weight matrix between the input $v_{i}$ and the hidden variable $h_{j}$, and $b$ and $c$ are model parameters. Generally speaking, RBMs can be viewed as estimators of the distribution underlying the training data (see equation III-A). The probability (distribution) assigned by the RBM configuration to every possible pair of visible and hidden vectors $(v, h)$ is given by the energy function. The joint distribution of the RBM configuration is

$$
\begin{aligned}
P(v, h) & =\frac{1}{Z} \exp (-\operatorname{Energy}(v, h)) \\
& =\frac{1}{Z} \prod_{i j} e^{W_{i j} v_{i} h_{j}} \prod_{i} e^{b_{i} v_{i}} \prod_{j} e^{a_{j} h_{j}},
\end{aligned}
$$

where $Z$ is a partition function denoted by $Z=$ $\sum_{v} \sum_{h} \exp (-\operatorname{Energy}(v, h))$.

Thus, DBN can be dened as a graphical model (see Figure 5), designed in an unsupervised manner with unlabeled training data. It is exploited to discover relevant features of data via multiple layers of nonlinear representations.

The principal building block of a DBN is an RBM [32], which represents a layer in the network. The training method proposed by [32] aims to train the whole deep network sequentially layer-by-layer, which means that each stacked RBM in the architecture is separately trained in an unsupervised manner. This approach is called greedy layer-wise, and it creates a synergy between the layers (RBMs) in that the output of one layer is the input of the layer. This gives DBNs the capability to discover features by learning a higher-level representation of the data through the succession of layers. Once the training phase is completed, each $k$-th RBM provides

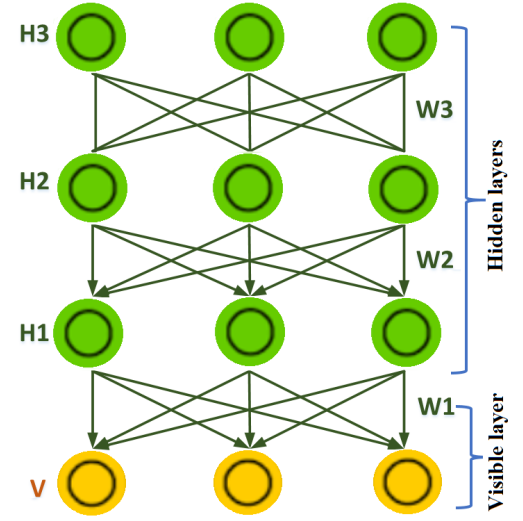

Fig. 5. A diagrammatic representation of a DBN model with three hidden layers.

a weight matrix $W_{k}$ with optional bias vectors for both the visible and hidden layers.

The DBN joint distribution between the observed vector $x$ and the $\ell$ hidden layers $h$ is given by [32]

$$
\begin{aligned}
P\left(x, h^{1}, h^{2}, \ldots, h^{l}\right)= & \left(P\left(x \mid h^{1}\right) P\left(h^{1} \mid h^{2}\right) \ldots P\left(h^{l-2} \mid h^{l-1}\right)\right) \\
& \times P\left(h^{\ell-1}, h^{\ell}\right),
\end{aligned}
$$

where $P\left(x \mid h^{1}\right)$ denotes the conditional distribution of the input $x$ and the first hidden layer $h^{1}, P\left(h^{1} \mid h^{2}\right)$ models the conditional distribution of hidden layer and each other as Sigmoid belief networks (see Figure 5). The term $P\left(h^{\ell-1}, h^{\ell}\right)$ expresses the joint distribution of an RBM. We expressed the DBN model in a compact formula:

$$
P\left(x, h^{1}, \ldots, h^{l}\right)=\left(\prod_{k=0}^{\ell-2} P\left(h^{k} \mid h^{k+1}\right)\right) P\left(h^{\ell-1}, h^{\ell}\right),
$$

where $x=h^{0}$ and $P\left(h^{k} \mid h^{k+1}\right)$ is a visible given hidden conditional distribution in an RBM corresponding to level $k$ of the DBN and expressed by $P\left(h^{k} \mid h^{k+1}\right)=\operatorname{sigmoid}\left(b^{k}+\right.$ $\left.W^{k} h^{k+1}\right)$, and $P\left(h^{\ell-1}, h^{\ell}\right)$ represents the joint distribution in the top-level RBM.

\section{B. One-class support vector machines (OCSVM)}

OCSVM can be regarded as a special case of the multiclass SVM scheme that separates normal from abnormal datasets [33]. In other words, OCSVM is constructed using only data from one class (i.e., the normal data). It is trained to build boundaries (hyperplanes) in an unsupervised manner based on training data. Generally speaking, the constructed boundaries are employed to check whether new data points have close features as the nominal class in the feature space $\mathcal{F}$ for normal observations, which is delimited by the hyperplanes. The decision process is performed via a decision function, $f(x)$, which gives the response +1 (inlier) if the tested data is within the feature space $\mathcal{F}$ of normal data and -1 (outlier) for observations classified as outside the feature space $\mathcal{F}$ (i.e., an anomaly). 
We let $x_{1}, \ldots, x_{j} \in \mathcal{D}$ and $j \in[1, k]$ be the training dataset. OCSVM maps input data into the high-dimensional feature space $\mathcal{F}$ via kernels such as the radial basis function (RBF) (see equation 6). As illustrated in Figure 6, the decision rule $f(x)$ aims to maximize the distance between the hyperplane $\mathcal{H}$, which separates the training data in the features space $\mathcal{F}$, and the origin. So, the objective function $f(x)$ is expressed as where $W, \rho$ and $\Psi$ represents respectively a weight vector,

$$
f(x)=\operatorname{sgn}((W \cdot \Psi(x))-\rho),
$$

an offset, and a feature map $\mathcal{D} \rightarrow \mathcal{F}$. $W$ and $\rho$ can be determined easily by solving the following quadratic optimization problem.

$$
\begin{aligned}
& \min _{w \in \mathcal{F}, \xi \in R^{l}, \rho \in R} \frac{1}{2}\|w\|^{2} \frac{1}{\nu l} \sum_{i}^{l} \xi_{i}-\rho, \\
& \text { s.t. to }(w . \Psi(x)) \geq \rho-\xi_{i}, \xi_{i} \geq 0,
\end{aligned}
$$

where $\nu \in[0,1]$ represents the parameter that defines the solution.

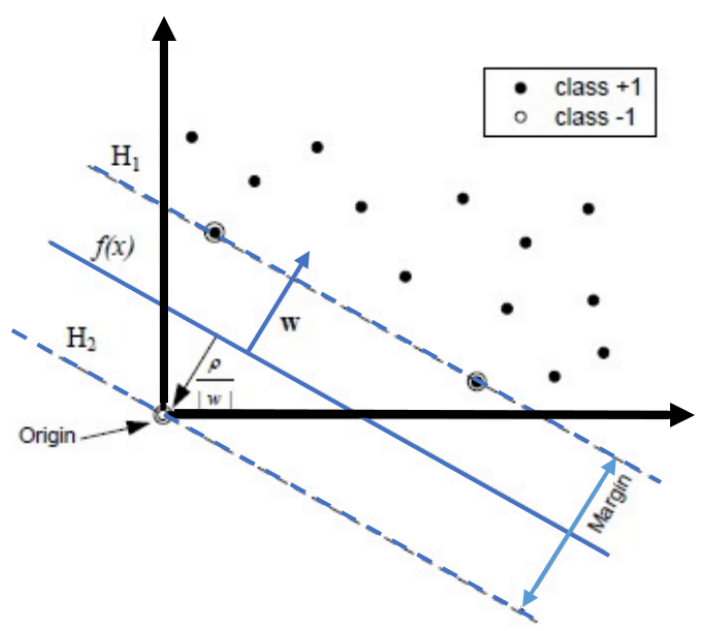

Fig. 6. Illustration of a one-class CSVM scheme.

\section{DBN-OCS VM METHODOLOGY}

Here, we describe the designed anomaly detection method that we will use for monitoring ozone measurements. Because model-based methods are not commonly available to model ozone variations due to its complex nonlinear dynamics, we adopted the DBN model, which is a powerful tool with greedy learning features, accounts for the nonlinear aspects of ozone variations. This methodology merges the benefits of a DBN modeling and anomaly detection ability of OCSVM to improve ozone monitoring (see Figure 8). DBNs exhibit high performance in identifying features from high-dimensional data [34]. Here, we first construct a DBN model with four RBM layers based on anomaly-free data (under anomaly-free conditions). The first layer is trained with training input data. Incorporating more hidden units to a given layer increases the flexibility of the model to extract relevant features from the analyzed data. After selecting the parameters of a given layer, which provide a minimum deviation between output and input, then its output will be the input of the next layer. In the DBN modeling, the features produced by the first layer are used to feed the second layer to obtain new features that will feed the next layer and so on. At the end of each layer, the model (i) discovers and extracts new features. After each model (ii), a new encoded output is generated that is used as input for the next layer. This greedy layer-wise training procedure has been proposed by [35] for building a DBN model.

Generally speaking, an appropriate model is able to extract the complexity features to mimic the process dynamics and approximatively reconstruct the original input data. In training stage, the designed deep learning model is usually validated by evaluating the reconstruction error. Indeed, cross entropy function has been commonly used to validate the quality of the built model. It measures the dissimilarity between two distributions of input and reconstructed data form the designed model. The cross-entropy function becomes closer to zero when the selected model describes well the input data. It is defined as [36]

$$
\mathcal{C}(X, \hat{X})=-\sum_{i}^{n}\left(\hat{X}_{i} \log \left(X_{i}\right)+\left(1-\hat{X}_{i}\right) \log \left(1-X_{i}\right)\right),
$$

where $X$ is the distribution of input data and $\hat{X}$ is the distribution of reconstructed data from the constructed model. The initialization of parameters plays a key role in reaching the global minimum of the cost function. Three main initializations methods are usually used to set the hyperparameters in deep learning architectures [37]: (1) random initialization, which randomly selects values of the starting parameters, (2) grid search which defines a range of hyperparameters and iterates over those ranges till reaching an acceptable result, and finally (3) the manual search based on knowledge of a problem in hand.

After constructing a reference DBN model, in the second phase, an OCSVM algorithm with a nonlinear kernel is trained with the features extracted by DBN. As stated above, DBN and OCSVM are both trained entirely in an unsupervised way. Then, the constructed DBN model is utilized with OCSVM to test new datasets. The main steps of the proposed approach are summarized in Algorithm 1. We apply this model to

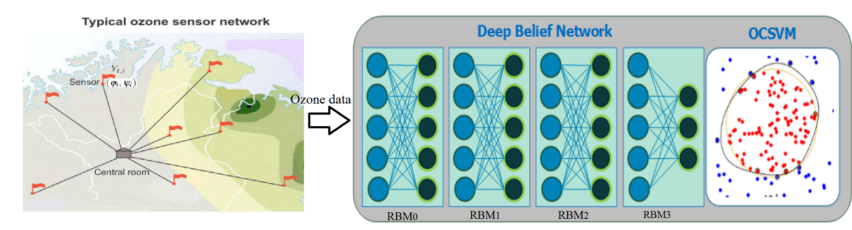

Fig. 7. Diagrammatic illustration of DBN-based OCSVM methodology.

inspect process variables for abnormal events by looking for future deviations from normality. In this approach, the output DBN model is used by the OCSVM algorithm for anomaly detection. A schematic illustration of the proposed DBNOCSVM strategy is given in Figure 8 and outlined below. 


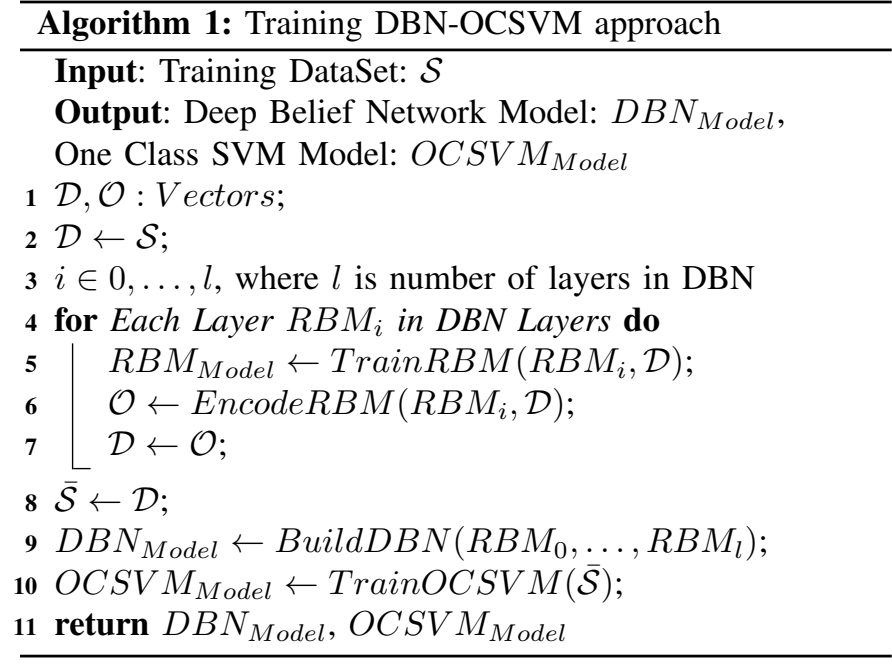

\section{Offline modeling}

Step (a):

1) Collection of data without anomalies (i.e., ambient pollution) to build training dataset $\mathcal{S}$.

2) Normalization of training dataset $\mathcal{S}$.

3) Greedy layer-wise unsupervised training of each layer (RBM) separately.

4) Hierarchical nonlinear transformations to discover features.

5) Building DBN model $\mathcal{M}$, weights matrix $\mathcal{W}$, and bias $\mathcal{B}$.

6) Generation of new training dataset $\overline{\mathcal{S}}$, which is an encoded version of the original dataset $\mathcal{S}$ that provides consistent features.

7) Unsupervised training of OCSVM using dataset $\overline{\mathcal{S}}$.

8) Building of OCSVM model for anomaly detection using hyperplanes.

\section{Online detection}

Step (a):

1) Acquisition and normalization of new observation $X$.

2) Encoding of the new observation $X$ using DBN model.

3) Classification of $X$ as inlier or outlier based on the OCSVM model hyperplanes.

\section{OZONE POLLUTION MONITORING}

This section is dedicated to an evaluation of the proposed anomaly-detection strategy with real datasets from Isére in France. The ozone data was downloaded from Atmo Auvergne-Rhône-Alpes's website (http://www.atmoauvergnerhonealpes.fr). Ozone data measured January 1st to March 4th, 2015 (see Figure 9), were used to develop a DBN model. Before we evaluate the performance of the proposed algorithm, we first conduct a descriptive analysis of the anomaly-free training data (of ambient ozone pollution). Figure 9 clearly shows that the variations in the ozone time series collected from different sensors behave similarly to each other.

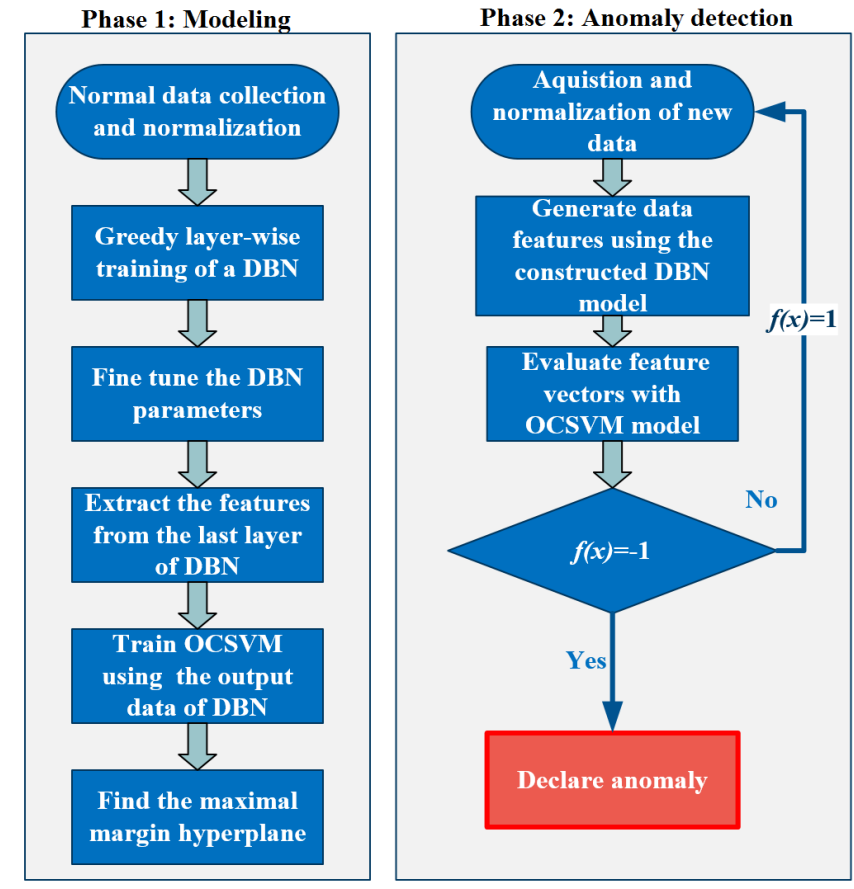

Fig. 8. DBN-based OCSVM fault detection methodology.

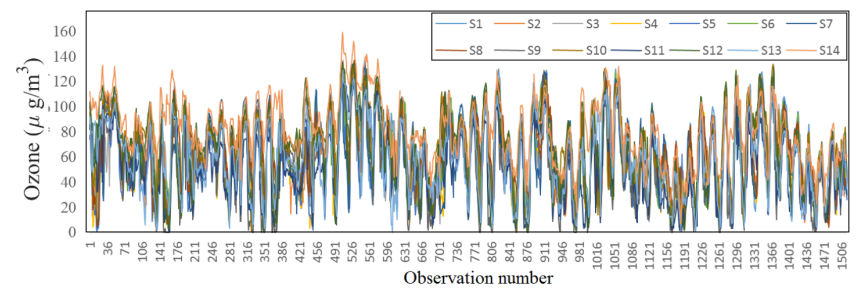

Fig. 9. Ozone measurements used in training.

Autocorrelation function (ACF) plots of the training time series from Figure 9 are presented in Figure 10. Figure 10 indicates an ACFperiodicity of 24 hours. One period is defined as the time between two successive maxima in the ACF (Figure 10). This periodicity is due to the diurnal ozone cycle which is mainly resulted from the diurnal temperature cycle.

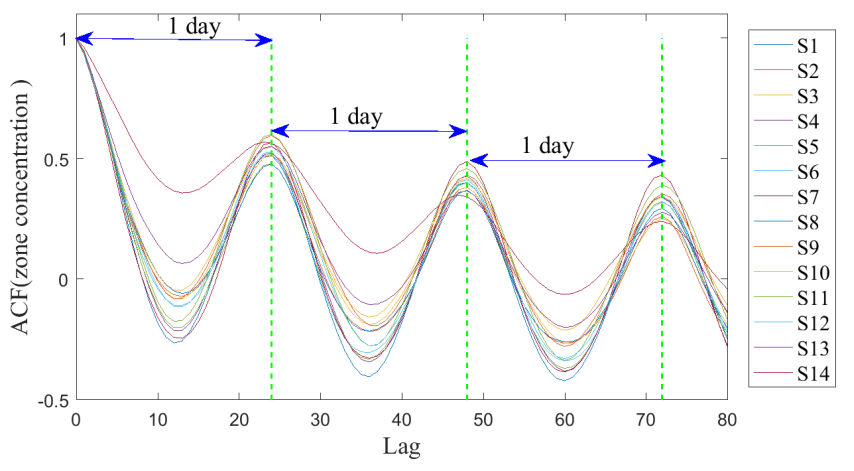

Fig. 10. Autocorrelation function of ozone data.

The summary statistics for the training dataset are shown in Table II. The location of the distribution of each variable (i.e., ozone measurements from each station) is captured by 
the mean value, while the spread in the dataset is delineated by standard deviations, extremes, and quartiles. The skewness and kurtosis represent the symmetry and flatness of the distribution of the studied time series. The kurtosis is used to quantify the non-Gaussianity of the data. The kurtosis, or the degree of peakedness of a distribution, is 3 for a Gaussian distribution. If the kurtosis is greater than 3, the distribution is superGaussian, i.e, more peaked than Gaussian; if it is less than 3, then the distribution is sub-Gaussian, i.e., flatter than Gaussian. Skewness indicates the asymmetry of the data compared to the sample mean. The skewness of a symmetric distribution, such as Gaussian, is zero. Negative skewness indicates that the distribution is skewed to the left side, and positive skewness indicates that the distribution is skewed to the right. From Table II, we conclude that the ozone data is approximately Gaussian (i.e., small values of skewness, and the kurtosis values are around 3 ).

For the training data in Figure 9, the box plots are shown in Figure 11. We see from Figure 11 that the data distribution from all sensors are almost symmetric and have comparable behavior.

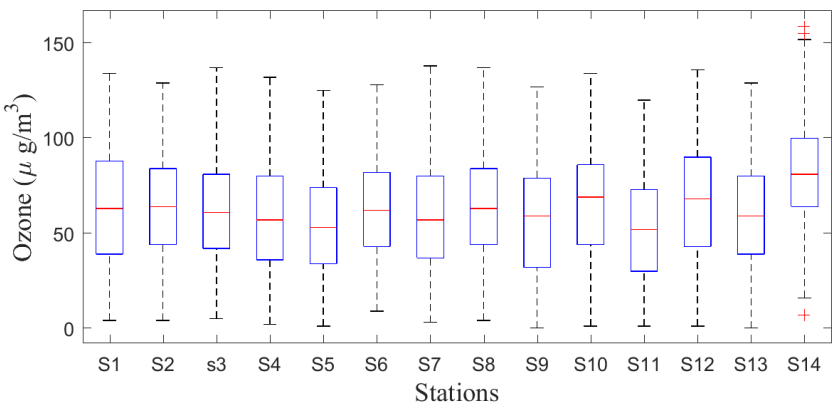

Fig. 11. The box plots of the data in Figure 9 .

The values of parameters of the designed DBN model and OCSVM scheme are given in Table III. After constructing the reference DBN model with anomaly-free data, we use the output features from DBN to monitor the abnormal ozone pollution with OCSVM.

In this study, the initial values of the DBN parameters are fixed manually during the training phase. A loss function is used to measure the error made by the network when trying to reproduce the input. This function is also called cost, objective or usually regularization term. As pointed out above, the crossentropy error function is used to quantify the quality of the designed model. The training is performed until the crossentropy error converges close to zero (closer to the global optimum). Figure 12 shows the convergence of the crossentropy loss when the number of epochs is around 180.

Abnormalities in ozone measurements can be categorized into two types: true and false anomalies. True anomalies are anomalies generated by an increase of ozone concentration (i.e., abnormal pollution) (Figure 13 (Left)). They are generated under certain conditions including sunny days under stagnant, humid air conditions and high temperatures to enhance the formation of ozone [17]. These anomalies are with a duration of several hours because of time required for

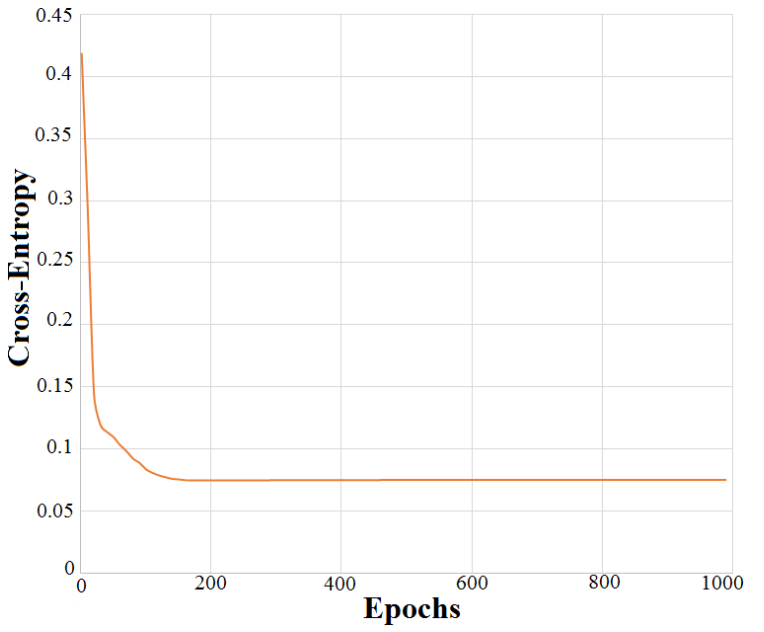

Fig. 12. The evolution of the cross-entropy error in function of the number of epochs during the training phase.

a progressive production of the photochemical ozone. While false anomalies are mainly related to sensors malfunctioning (i.e., sensor drifts) and are very short with a duration less than an hour and are very large (Figure 13 (Right)).
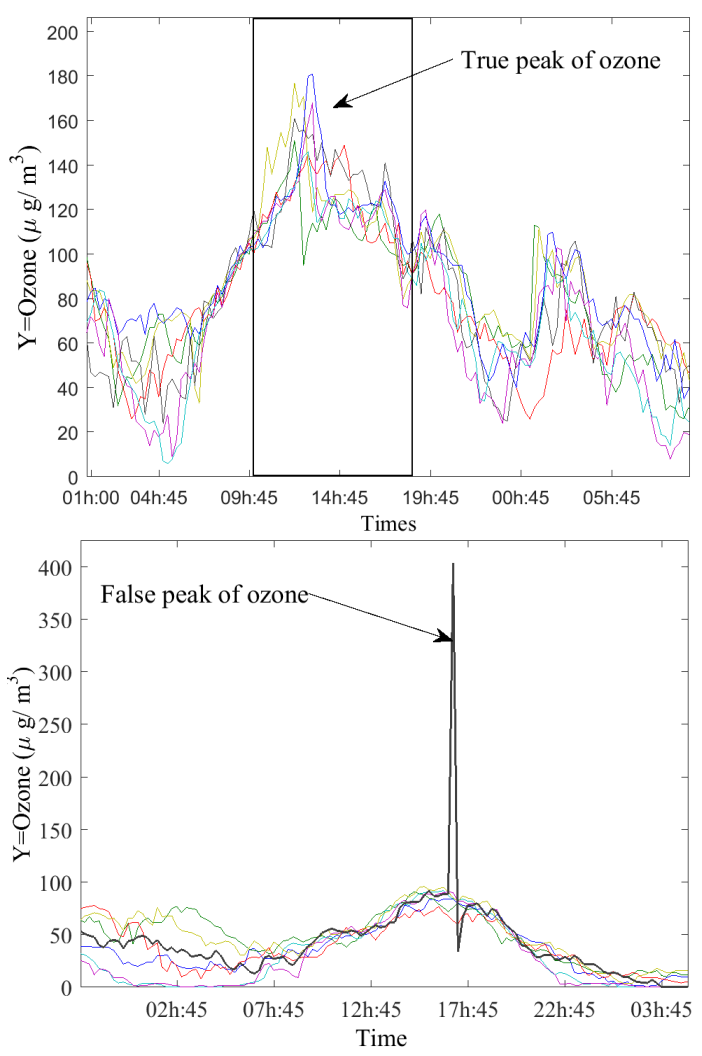

Fig. 13. (Top) Photochemical ozone pollution and (Bottom) spike in ozone concentration due to sensors malfunction.

To detect atypical ozone peaks, we complete two steps. First, we design a model that describes the variation in ozone pollution. Then, based on the DBN model and the OCSVM detector, we check the new measured data for anomalies. We combine the DBN model with the OCSVM detector using an 
TABLE II

DESCRIPTIVE STATISTICS OF THE TRAINING DATASET.

\begin{tabular}{llllllllll}
\hline & Mean & STD & Min & 1st Quartile & Median & 3rd Quartile & Max & Skewness & Kurtosis \\
\hline S1 & 63.80 & 30.85 & 4.00 & 39.00 & 63.00 & 88.00 & 134.00 & 0.08 & 2.07 \\
S2 & 63.89 & 26.51 & 4.00 & 44.00 & 64.00 & 84.00 & 129.00 & 0.10 & 2.33 \\
S3 & 61.96 & 26.12 & 5.00 & 42.00 & 61.00 & 81.00 & 137.00 & 0.15 & 2.39 \\
S4 & 58.75 & 29.06 & 2.00 & 36.00 & 57.00 & 80.00 & 132.00 & 0.18 & 2.26 \\
S5 & 54.99 & 26.86 & 1.00 & 34.00 & 53.00 & 74.00 & 125.00 & 0.26 & 2.33 \\
S6 & 63.20 & 26.41 & 9.00 & 43.00 & 62.00 & 82.00 & 128.00 & 0.24 & 2.32 \\
S7 & 59.71 & 28.20 & 3.00 & 37.00 & 57.00 & 80.00 & 138.00 & 0.36 & 2.37 \\
S8 & 64.33 & 27.38 & 4.00 & 44.00 & 63.00 & 84.00 & 137.00 & 0.09 & 2.36 \\
S9 & 55.40 & 31.03 & 0 & 32.00 & 59.00 & 79.00 & 127.00 & -0.07 & 2.12 \\
S10 & 65.75 & 29.10 & 1.00 & 44.00 & 69.00 & 86.00 & 134.00 & -0.16 & 2.37 \\
S11 & 52.88 & 28.31 & 1.00 & 30.00 & 52.00 & 73.00 & 120.00 & 0.16 & 2.23 \\
S12 & 66.29 & 31.20 & 1.00 & 43.00 & 68.00 & 90.00 & 136.00 & -0.13 & 2.21 \\
S13 & 59.11 & 27.40 & 0 & 39.00 & 59.00 & 80.00 & 129.00 & 0.01 & 2.21 \\
S14 & 81.84 & 24.83 & 7.00 & 64.00 & 81.00 & 100.00 & 159.00 & 0.10 & 2.68 \\
\hline
\end{tabular}

TABLE III

PARAMETER VALUES OF THE DBN MODEL AND OCSVM SCHEME.

\begin{tabular}{l|ll} 
Models & Parameter & Value \\
\hline & Layers & 3 \\
DBN & Units & 17 \\
& Learning rate & 0.001 \\
& Epochs & 1000 \\
\hline OCSVM & Kernel & RBF \\
RBF & $\gamma$ & 0.1 \\
RBF & $\nu$ & 0.001 \\
\hline
\end{tabular}

RBF kernel and parameters values $(\gamma=0.1$ and $\nu=0.001)$. We compare the efficiency of the designed methodology to that declared by the experts of Atmo Auvergne-Rhône-Alpes. The testing measurements comprise the period between January 1st and September 10th, 2017. The detection results are given in Table IV.

\begin{tabular}{|l|l|l|l|l|l|}
\hline Day & Month & Year & Alert & Period & DBN-OCSVM \\
\hline 29 & August & 2017 & Pollution vigilance & $(13 \mathrm{~h}-20 \mathrm{~h})$ & Yes \\
\hline 4 & August & 2017 & Pollution vigilance & $(13 \mathrm{~h}-20 \mathrm{~h})$ & Yes \\
\hline 3 & August & 2017 & Pollution vigilance & $(13 \mathrm{~h}-20 \mathrm{~h})$ & Yes \\
\hline 7 & July & 2017 & Pollution vigilance & $(13 \mathrm{~h}-20 \mathrm{~h})$ & Yes \\
\hline 23 & June & 2017 & Pollution vigilance & $(13 \mathrm{~h}-20 \mathrm{~h})$ & Yes \\
\hline 22 & June & 2017 & Pollution vigilance & $(13 \mathrm{~h}-20 \mathrm{~h})$ & Yes \\
\hline 20 & June & 2017 & Pollution vigilance & $(13 \mathrm{~h}-20 \mathrm{~h})$ & Yes \\
\hline
\end{tabular}

DETECTION RESULTS FROM THE DBN-OCSVM ALGORITHM.

The ozone data with atypical ozone measurements that occurred on June 22nd and 23rd, 2017 and the corresponding DBN-OCSVM results are demonstrated in plots (a) and (b) of Figure 14. The output of DBN-OCSVM is ' 1 ' in the case of normal measurements and ' -1 ' in the case of anomalies in ozone data. We see that the DBN-based OCSVM chart detects the abnormal ozone measurements.

The proposed approach is running entirely using a single CPU desktop machine with Intel i7 4770 CPU based on Streaming SIMD Extensions (SSE) technology. This approach is fast and provides the detection result in around $10 \mathrm{~ms}$

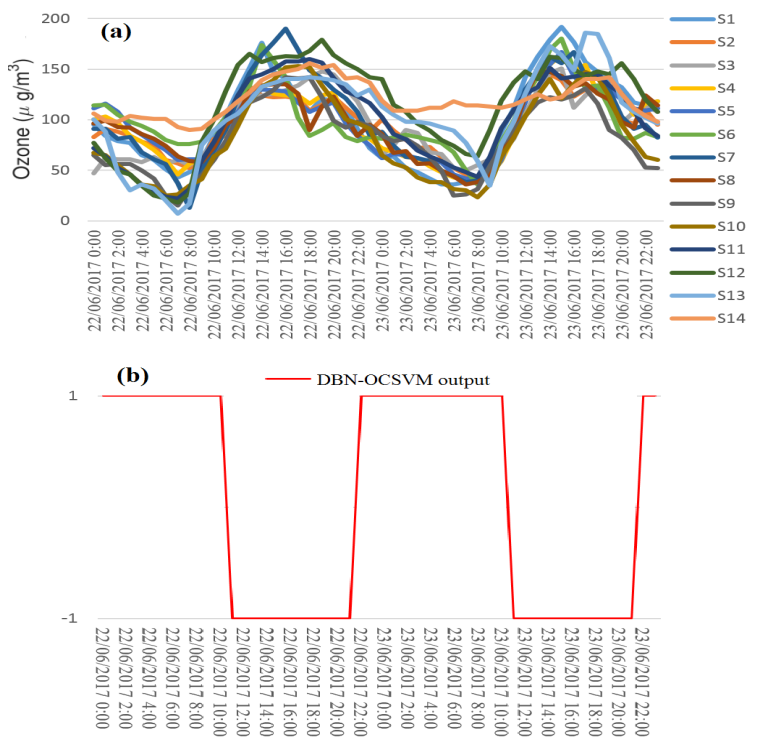

Fig. 14. Original ozone data (plot(a)). The DBN-OCSVM chart (plot(b)) in the case with abnormal measurements occurred on 22 and 23 June 2017.

running time, which is acceptable and meets the requirement of the real-time application.

\section{A. Sensor anomaly detection: False anomalies}

In this section, we assess the performance of the DBNOCSVM algorithm in detecting sensor faults (i.e., faults in sensor readings). We note that high ozone concentrations related to sensor faults can be observed outside the summer period and even in a night, which is not the case for ozone pollution produced via photochemical ozone. Also, sensor faults may produce unusually high values, from 150 to over $600 \mathrm{\mu g} / \mathrm{m}^{3}$. Here, we inject three types of faults to the testing datasets and assess the efficiency of the proposed algorithm. In the first case study, we introduce a single bias fault (i.e., in one variable) to the testing data from $S 2$, where the measured values are shifted by a bias, $x(t)+b$, from the correct measurements. In the second case study, we simultaneously inject multiple bias faults into the data from $S 3$ and $S 6$. In the third case study, we introduce an intermittent fault into the data of S5. Here, 


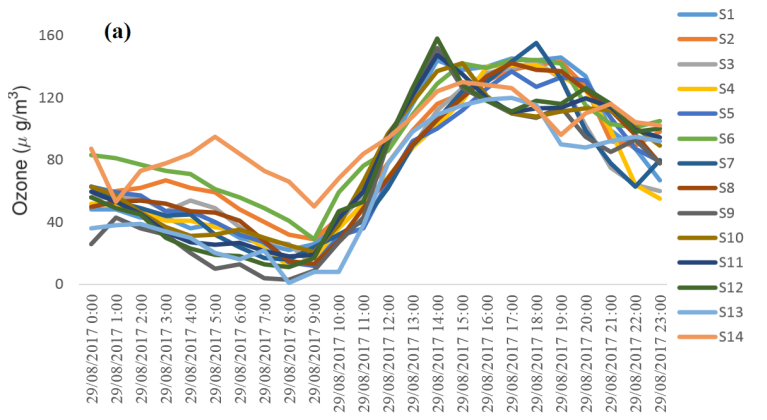

(b)

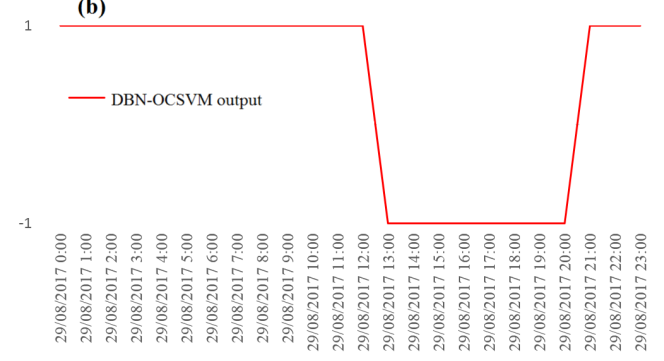

Fig. 15. Original ozone data (plot(a)). The DBN-OCSVM scheme (plot(b)) in the case with abnormal measurements on 29 August 2017.

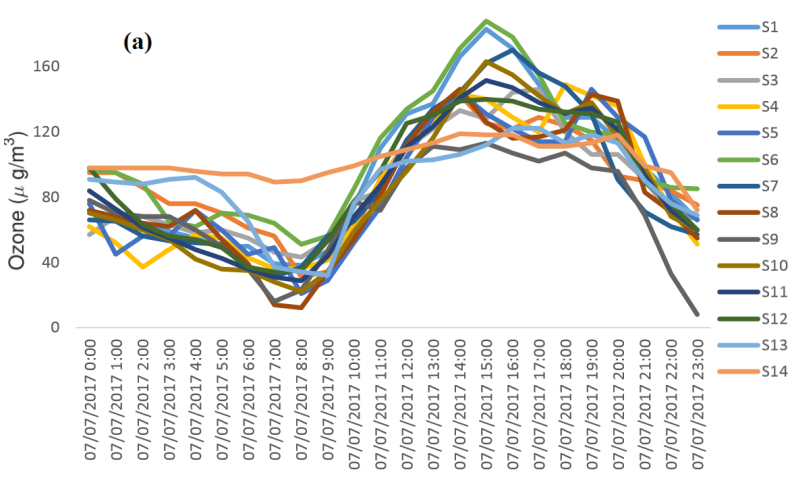

(b)

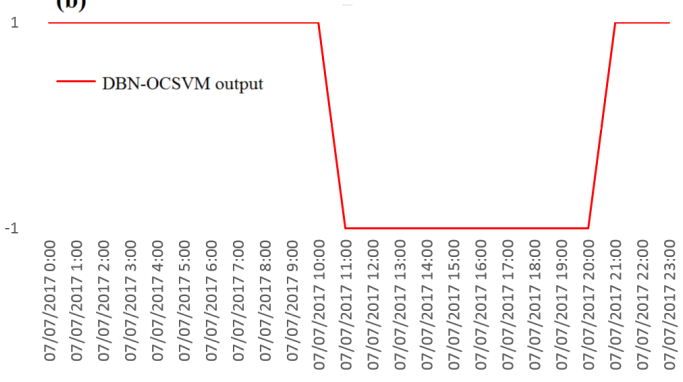

Fig. 16. Original ozone data (plot(a)). The DBN-OCSVM scheme (plot(b)) in the case with abnormal measurements on July 7th, 2017.

we utilize the Area Under Curve (AUC) metric to evaluate the accuracy of the proposed algorithm. An AUC of 1 corresponds to an ideal detection, and an $A U C>0.9$ corresponds to a good detection. In this section, we present a comparison between the proposed approach DBN3-OCSVM and that of the RBM, DBN2, and deep stacked autoencoder (DSA) [38]-based OCSVM methods and DBN-based Expectation Maximization (EM) [39], Birch [40] and K-means [41] approaches. Here, DBN2 comprises two hidden layers and proposed DBN model comprises three layers. The experimental parameters of the studied algorithms are presented in Table V.

TABLE V

VALUES OF PARAMETERS USED IN THE STUDIED SCHEMES.

\begin{tabular}{l|ll}
\hline Models & Parameter & Value \\
\hline \multirow{3}{*}{ Autoencoder } & weights & uniform \\
& Learning rate & 0.01 \\
& Training epochs & 100 \\
\hline Birch & branch & 50 \\
\hline \multirow{3}{*}{ K-means } & init & k-means++ \\
& init & 10 \\
& iteration & 300 \\
\hline \multirow{3}{*}{ EM } & covarianceType & full \\
& covar & $1 \mathrm{e}-06$ \\
& iteration & 100 \\
\hline
\end{tabular}

1) Case A: Single abrupt fault: Here, we evaluate the performance of the DBN-OCSVM algorithm in detecting a single bias sensor fault. We introduced the bias to measurements collected by $S_{2}$. Then, we apply the DBN-OCSVM algorithm and compute the AUC values for each fault magnitude between $5 \%$ and $100 \%$ of the total variation found in the raw data. Figure 17 shows the AUC values of DBN3, DBN2, RBM and DSA-based OCSVM algorithms, and standalone OCSVM for different fault magnitudes.

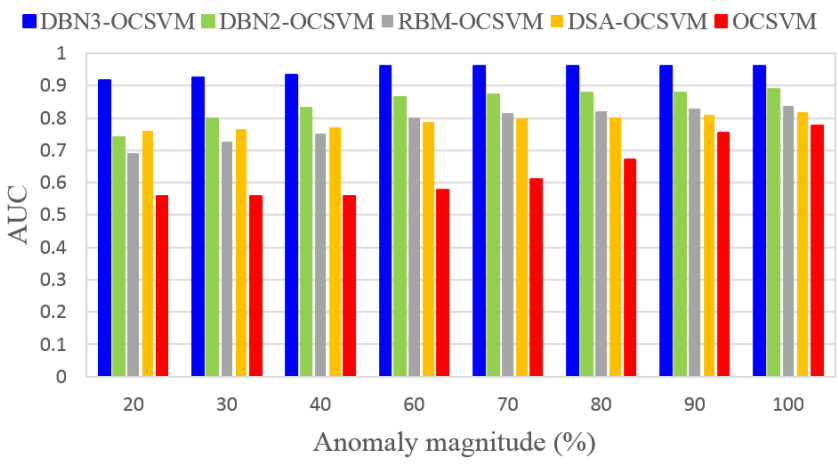

Fig. 17. AUC comparison of the studied fault detection methods (i.e., DBN3, DBN2, DBN1RBM, and DSA-based OCSVM and standalone OCSVM methods) for different fault magnitudes (case A).

From Figure 17, we see that the DBN-OCSVM algorithm performs well in detecting the single bias fault, even when the magnitude is relatively small. Results in Figure 17 confirms also the superiority of the DBN-OCSVM approach compared to RBM, DBN2 and DSA-based OCSVM and standalone OCSVM algorithms. These results indicate that deeper DBN architecture with three layers provides superior performance compared to RBM and DBN2-based OCSVM approaches. Based on this result, the selected DBN model with three layers provides more accurate detection compared to the RBM and DBN2. As a matter of fact, including more layers to the network may increase the capability of the model to extract relevant features from the input data. Also, we compared the integrated DBN-OCSVM to that of the standalone OCSVM and found better performance in detecting abnormalities in ozone data. Compared with DSA-OCSVM, the proposed approach delivers high detection performance. This is mainly 
due to the flexibility and efficiency of DBN model to extract the important features from the data and the high capacity of OCSVM to quantitatively discriminate between abnormal and normal features.

We also compared the detection efficiency of the proposed approach to that of DBN-based EM, K-means, and Birch algorithms (Figure 18). Here, these clustering schemes are applied to the features from the last layer in the DBN model. Figure 18 indicates that the DBN-OCSVM has surpassed DBN-based EM, K-means, and Birch algorithms. OCSVM algorithm is designed without considering assumptions of data convexity as in EM algorithm. In OCSVM algorithm, a kernel function projects the input data to a higher dimensional space to make a clear separation between normal and abnormal features. In addition, OCSVM is insensitive to the rank of the data record as the case for Birch scheme. Unsupervised OCSVM is easy to implement and requires only the faultfree data for training without the need for any labeled data. Of course, the proposed technique showed high sensitivity to abrupt changes in ozone measurements compared to the other studied approaches.

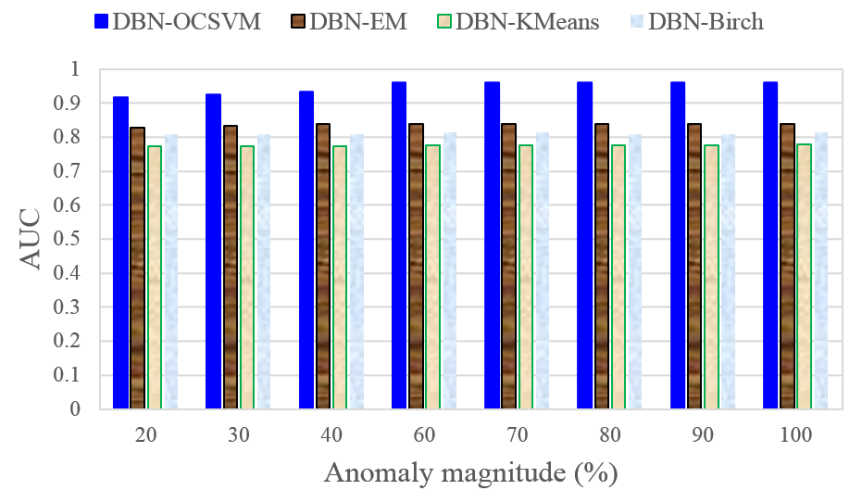

Fig. 18. AUC comparison of the DBN-based OCSVM, EM, K-means, and Birch methods for different fault magnitudes (case A).

2) Case B: Multiple abrupt faults: We simulate a bias sensor fault by simultaneously introducing a small constant change to the ozone measurements of both $S_{3}$ and $S_{6}$ between sample numbers 240 and 300 of the testing data. The AUC values of the DBN-OCSVM scheme with different numbers of hidden layers (i.e., RBM, DBN2 and DBN3), the DSAOCSVM and the standalone OCSVM algorithms computed for different fault magnitude values are presented in Figure 19. By comparing the RBM, DBN2, and DBN3-based OCSVM algorithms, we conclude that including more hidden layers to the DBN model enhance the fault detection performance. From the figure, we see that the DBN-OCSVM scheme performs well in detecting multiple faults (e.g., faults with $20 \%$ magnitude can be detected with AUC=0.916). Results in Figure 19 confirms the superiority of the DBN3-OCSVM approach compared to DSA-OCSVM and the standalone OCSVM. Unlike deep models, standalone OCSVM is less effective in detecting small and moderate anomalies in ozone measurements, i.e., its detection capability decrease in the case of small faults (e.g., faults with $20 \%$ magnitude can be detected with $\mathrm{AUC}=0.559)$. Thus, combining OCSVM algorithm with deep learning models improve its detection capacity.

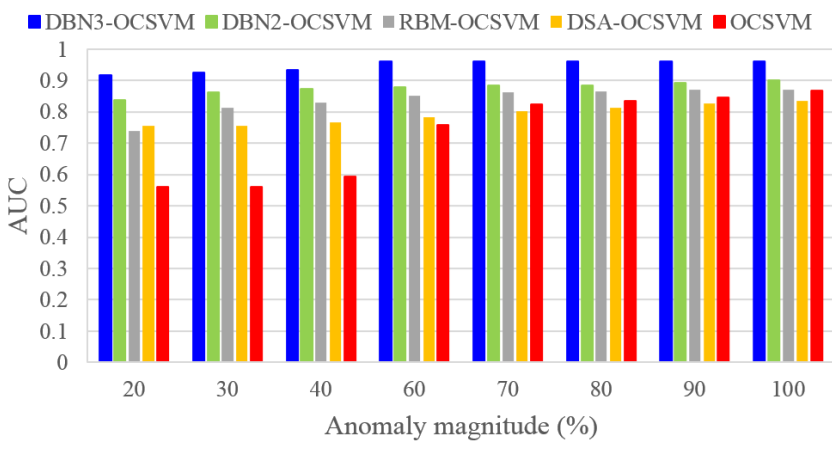

Fig. 19. AUC comparison of the studied fault detection methods (i.e., DBN3, DBN2, RBM, and DSA-based OCSVM and standalone OCSVM methods) for different fault magnitudes (case B).

Now, the performances of DBN-OCSVM approach in detecting multiple anomalies in multivariate ozone data are compared with DBN-based EM, K-means and Birch algorithm (Figure 20). Similar to the previous results, the detection results clearly indicate the superiority of DBN-OCSVM approach in detecting multiple anomalies in ozone data. Overall, the DBN-OCSVM showed the best detection quality in detecting abnormalities in ozone data.

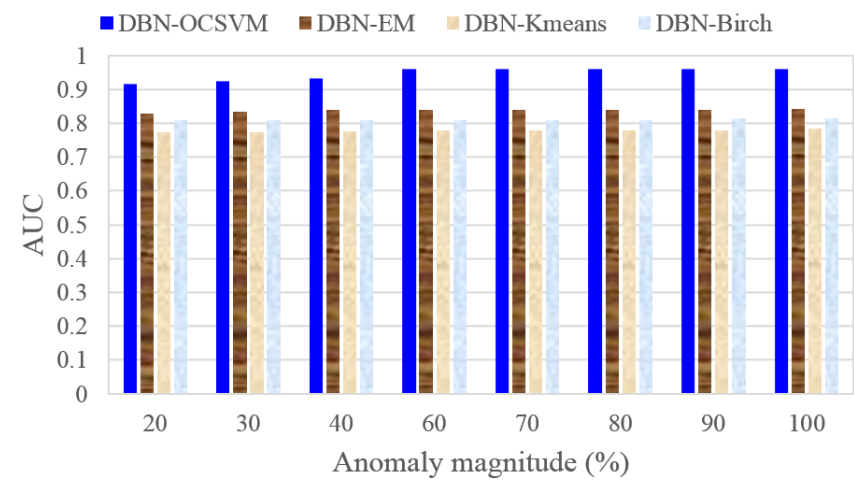

Fig. 20. AUC comparison of the DBN-based OCSVM, EM, K-means, and Birch methods for different fault magnitudes (case B).

3) Case C: Intermittent faults: Here, we assess the DBNOCSVM scheme in the presence of intermittent sensor faults that occur and disappear repeatedly. We introduce a simulated intermittent fault into the raw data of $S 5$. Specifically, we inject a bias in the ozone concentration measured by $S_{5}$ from samples 410 to 440 , and from samples 502 to 520 of the testing data. To evaluate the sensitivity of DBN-OCSVM to these intermittent faults, we change the amplitude of the bias fault and compute the AUC obtained by DBN3OCSVM, DBN2-OCSVM, RBM-OCSVM, DSA-OCSVM and standalone OCSVM algorithms. The AUC as a function of the fault magnitude is shown in Figure 21. We see that the DBN-OCSVM algorithm performs suitably well in detecting intermittent sensor faults. It detects intermittent faults with a magnitude of $20 \%$ of the total variation found in the raw data, with AUC around 0.89, which is very promising. Our study again testifies that the performance of the OCSVM chart 
improved when applied to features from DBN model than from the RBM, DBN2, and DSA.

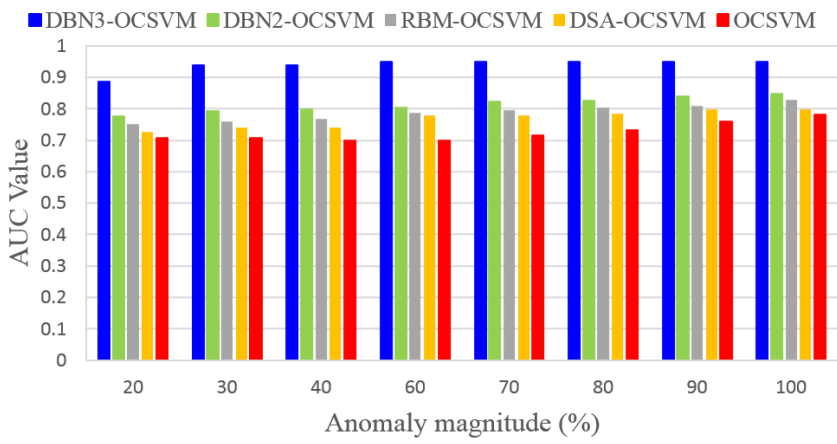

Fig. 21. AUC comparison of the studied fault detection methods (i.e., DBN3, DBN2, RBM, and DSA-based OCSVM and standalone OCSVM methods) for different fault magnitudes (case C).

The superiority of the DBN-OCSVM approach, in detecting intermittent anomalies in ozone measurements, over other charts can be confirmed by the results in Figure 22, showing that the DBN-OCSVM approach has the highest AUC values compared to the DBN-based EM, K-means and Birch approaches.

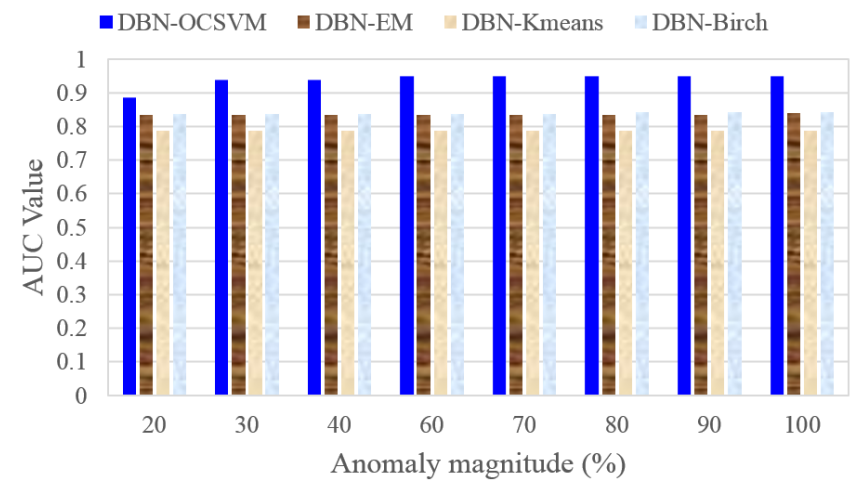

Fig. 22. AUC comparison of the DBN-based OCSVM, EM, K-means, and Birch methods for different fault magnitudes (case C).

Thus, our results demonstrate that the DBN-OCSVM algorithm performs reasonably well in detecting real ozone pollution and abnormal measurements resulting from sensor malfunctions. This study shows, in particular, the benefit of integrating a DBN model, which extracts the features of high dimensional data, with OCSVM, which differentiates the new data from the normal training data. Also, results demonstrated that the proposed DBN-OCSVM methodology shows a good performance compared to the DSA-based OCSVM and DBNbased EM, K-means, and Birch schemes.

\section{CONCLUSION}

Reliable monitoring of air quality is an essential element for improving air quality management and to ensuring a proper environment for good health and quality living. In this paper, an unsupervised anomaly detection strategy is designed for monitoring ozone measurements. This strategy integrates the DBN modeling with the sensitivity to small changes of OCSVM scheme. We first evaluated the efficiency of the developed algorithm to detect abnormal ozone pollution real data from Isère in France. The developed approach correctly detected all anomalies reported by the experts of Atmo Auvergne-Rhône-Alpes association. We also applied this approach to detect sensors anomalies. Furthermore, we also compared the efficiency of the proposed approach with RBM and DSA-based K-Means, EM and Birch algorithms. Results verify the ability of the DBN-OCSVM methodology to identify abnormalities in ozone measurements.

As future work, it would be interesting to incorporate more data inputs, such as meteorological data or other pollutants to enhance the efficiency of the proposed approach.

\section{ACKNOWLEDGEMENT}

The research reported in this publication was supported by funding from King Abdullah University of Science and Technology (KAUST) Office of Sponsored Research (OSR) under Award No: OSR-2015-CRG4-2582.

\section{REFERENCES}

[1] M. Bacco, F. Delmastro, E. Ferro, and A. Gotta, "Environmental monitoring for smart cities," IEEE Sensors Journal, vol. 17, no. 23, pp. 7767-7774, 2017.

[2] K. B. Shaban, A. Kadri, and E. Rezk, "Urban air pollution monitoring system with forecasting models," IEEE Sensors Journal, vol. 16, no. 8, pp. 2598-2606, 2016.

[3] K. S. E. Phala, A. Kumar, and G. P. Hancke, "Air quality monitoring system based on ISO/IEC/IEEE 21451 standards," IEEE Sensors Journal, vol. 16 , no. 12 , pp. 5037-5045, 2016.

[4] F. Harrou, L. Fillatre, and I. Nikiforov, "Bounded nuisance rejection and redundant sensor network," in International Conference, System Identification and Control Problems, SICPRO'09. Moscow, 2009, pp. 786-795.

[5] J.-Y. Kim, C.-H. Chu, and S.-M. Shin, "ISSAQ: An integrated sensing systems for real-time indoor air quality monitoring," IEEE Sensors Journal, vol. 14, no. 12, pp. 4230-4244, 2014.

[6] H. Moshammer, "Communicating health impact of air pollution," in Air Pollution. InTech, 2010.

[7] F. Biancofiore, M. Verdecchia, P. Di Carlo, B. Tomassetti, E. Aruffo, M. Busilacchio, S. Bianco, S. Di Tommaso, and C. Colangeli, "Analysis of surface ozone using a recurrent neural network," Science of the Total Environment, vol. 514, pp. 379-387, 2015.

[8] L. E. Plummer, S. Smiley-Jewell, and K. E. Pinkerton, "Impact of air pollution on lung inflammation and the role of toll-like receptors," International Journal of Interferon, Cytokine and Mediator Research, vol. 4, no. 1, pp. 43-57, 2012.

[9] O. Taylan, "Modelling and analysis of ozone concentration by artificial intelligent techniques for estimating air quality," Atmospheric Environment, vol. 150, pp. 356-365, 2017.

[10] J.-S. Hwang and C.-C. Chan, "Effects of air pollution on daily clinic visits for lower respiratory tract illness," American journal of epidemiology, vol. 155, no. 1, pp. 1-10, 2002.

[11] F. Harrou, M. Nounou, and H. Nounou, "Statistical detection of abnormal ozone levels using principal component analysis," ternational Journal of Engineering \& Technology, vol. 12, no. 6, pp. 54-59, 2012.

[12] M. P. Rissanen, T. Kurtén, M. Sipila, J. A. Thornton, J. Kangasluoma, N. Sarnela, H. Junninen, S. Jørgensen, S. Schallhart, M. K. Kajos et al., "The formation of highly oxidized multifunctional products in the ozonolysis of cyclohexene," Journal of the American Chemical Society, vol. 136, no. 44, pp. 15 596-15606, 2014.

[13] A. Nawahda, "An assessment of adding value of traffic information and other attributes as part of its classifiers in a data mining tool set for predicting surface ozone levels," Process Safety and Environmental Protection, vol. 99, pp. 149-158, 2016. 
[14] S. A. Abdul-Wahab, C. S. Bakheit, and S. M. Al-Alawi, "Principal component and multiple regression analysis in modelling of ground-level ozone and factors affecting its concentrations," Environmental Modelling \& Software, vol. 20, no. 10, pp. 1263-1271, 2005.

[15] C. Vlachokostas, S. Nastis, C. Achillas, K. Kalogeropoulos, I. Karmiris, N. Moussiopoulos, E. Chourdakis, G. Banias, and N. Limperi, "Economic damages of ozone air pollution to crops using combined air quality and GIS modelling," Atmospheric Environment, vol. 44, no. 28, pp. 3352-3361, 2010.

[16] C. Dueñas, M. Fernández, S. Canete, J. Carretero, and E. Liger, "Analyses of ozone in urban and rural sites in málaga (spain)," Chemosphere, vol. 56, no. 6, pp. 631-639, 2004.

[17] F. Harrou, F. Kadri, S. Khadraoui, and Y. Sun, "Ozone measurements monitoring using data-based approach," Process Safety and Environmental Protection, vol. 100, pp. 220-231, 2016.

[18] F. Harrou, A. Dairi, Y. Sun, and M. Senouci, "Reliable detection of abnormal ozone measurements using an air quality sensors network," in 2018 IEEE International Conference on Environmental Engineering (EE). IEEE, 2018, pp. 1-5.

[19] F. Harrou, L. Fillatre, M. Bobbia, and I. Nikiforov, "Statistical detection of abnormal ozone measurements based on constrained generalized likelihood ratio test," in Decision and Control (CDC), 2013 IEEE 52nd Annual Conference on. IEEE, 2013, pp. 4997-5002.

[20] Y.-D. Zhang, Y. Zhang, X.-X. Hou, H. Chen, and S.-H. Wang, "Sevenlayer deep neural network based on sparse autoencoder for voxelwise detection of cerebral microbleed," Multimedia Tools and Applications, vol. 77, no. 9, pp. 10 521-10538, 2018.

[21] W. Jia, M. Yang, and S.-H. Wang, "Three-category classification of magnetic resonance hearing loss images based on deep autoencoder," Journal of medical systems, vol. 41, no. 10, p. 165, 2017.

[22] Y. Jia, J. Wu, and Y. Du, "Traffic speed prediction using deep learning method," in Intelligent Transportation Systems (ITSC), 2016 IEEE 19th International Conference on. IEEE, 2016, pp. 1217-1222.

[23] D. Ravì, C. Wong, F. Deligianni, M. Berthelot, J. Andreu-Perez, B. Lo, and G.-Z. Yang, "Deep learning for health informatics," IEEE journal of biomedical and health informatics, vol. 21, no. 1, pp. 4-21, 2017.

[24] O. Costilla-Reyes, P. Scully, and K. B. Ozanyan, "Deep neural networks for learning spatio-temporal features from tomography sensors," IEEE Transactions on Industrial Electronics, 2017.

[25] A. Koesdwiady, R. Soua, and F. Karray, "Improving traffic flow prediction with weather information in connected cars: A deep learning approach," IEEE Transactions on Vehicular Technology, vol. 65, no. 12, pp. 9508-9517, 2016.

[26] J. Wang, X. Zhang, Q. Gao, H. Yue, and H. Wang, "Device-free wireless localization and activity recognition: A deep learning approach," IEEE Transactions on Vehicular Technology, vol. 66, no. 7, pp. 6258-6267, 2017.

[27] X. Wang, L. Gao, S. Mao, and S. Pandey, "CSI-based fingerprinting for indoor localization: A deep learning approach," IEEE Transactions on Vehicular Technology, vol. 66, no. 1, pp. 763-776, 2017.

[28] J. H. Seinfeld and S. N. Pandis, Atmospheric chemistry and physics: from air pollution to climate change. John Wiley \& Sons, 2016.

[29] B. Özbay, G. A. Keskin, Ş. Ç. Doğruparmak, and S. Ayberk, "Multivariate methods for ground-level ozone modeling," Atmospheric Research, vol. 102 , no. 1-2, pp. 57-65, 2011

[30] A. Dairi, F. Harrou, M. Senouci, and Y. Sun, "Unsupervised obstacle detection in driving environments using deep-learning-based stereovision," Robotics and Autonomous Systems, vol. 100, pp. 287-301, 2018.

[31] A.-r. Mohamed, G. E. Dahl, and G. Hinton, "Acoustic modeling using deep belief networks," IEEE Transactions on Audio, Speech, and Language Processing, vol. 20, no. 1, pp. 14-22, 2012.

[32] G. E. Hinton, S. Osindero, and Y.-W. Teh, "A fast learning algorithm for deep belief nets," Neural computation, vol. 18, no. 7, pp. 1527-1554, 2006.

[33] B. Schölkopf, J. C. Platt, J. Shawe-Taylor, A. J. Smola, and R. C. Williamson, "Estimating the support of a high-dimensional distribution," Neural computation, vol. 13, no. 7, pp. 1443-1471, 2001.

[34] Y. Bengio, P. Lamblin, D. Popovici, and H. Larochelle, "Greedy layerwise training of deep networks," in Advances in neural information processing systems, 2007, pp. 153-160.

[35] G. E. Hinton, S. Osindero, and Y.-W. Teh, "A fast learning algorithm for deep belief nets," Neural computation, vol. 18, no. 7, pp. 1527-1554, 2006.

[36] P. Vincent, H. Larochelle, I. Lajoie, Y. Bengio, and P.-A. Manzagol, "Stacked denoising autoencoders: Learning useful representations in a deep network with a local denoising criterion," Journal of Machine Learning Research, vol. 11, no. Dec, pp. 3371-3408, 2010.
[37] Y. Bengio, "Practical recommendations for gradient-based training of deep architectures," in Neural networks: Tricks of the trade. Springer, 2012, pp. 437-478.

[38] Y. Bengio et al., "Learning deep architectures for AI," Foundations and trends $(\mathrm{R}$ in Machine Learning, vol. 2, no. 1, pp. 1-127, 2009.

[39] A. P. Dempster, N. M. Laird, and D. B. Rubin, "Maximum likelihood from incomplete data via the em algorithm," Journal of the royal statistical society. Series B (methodological), pp. 1-38, 1977.

[40] T. Zhang, R. Ramakrishnan, and M. Livny, "Birch: an efficient data clustering method for very large databases," in ACM Sigmod Record, vol. 25, no. 2. ACM, 1996, pp. 103-114.

[41] D. Arthur and S. Vassilvitskii, "k-means++: The advantages of careful seeding," in Proceedings of the eighteenth annual ACM-SIAM symposium on Discrete algorithms. Society for Industrial and Applied Mathematics, 2007, pp. 1027-1035. 